Cipango Cahiers d'études japonaises

19 | 2012

Le Japon et le fait colonial II

\title{
Japon-Corée, France-Algérie
}

Réflexions sur deux situations coloniales et postcoloniales

Japan-Korea, France-Algeria. Some Reflections on Two Colonial and Postcolonial Situations

\section{Lionel Babicz}

\section{(2) OpenEdition Journals}

\section{Édition électronique}

URL : https://journals.openedition.org/cipango/1692

DOI : 10.4000/cipango.1692

ISSN : 2260-7706

\section{Éditeur}

INALCO

Édition imprimée

Date de publication : 30 octobre 2012

Pagination : 55-74

ISBN : 978-2-85831-204-7

ISSN : $1164-5857$

\section{Référence électronique}

Lionel Babicz, « Japon-Corée, France-Algérie », Cipango [En ligne], 19 | 2012, mis en ligne le 27 mars 2014, consulté le 30 juin 2021. URL : http://journals.openedition.org/cipango/1692 ; DOI : https:// doi.org/10.4000/cipango.1692

Ce document a été généré automatiquement le 30 juin 2021.

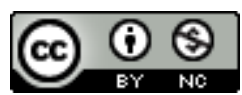

Cipango est mis à disposition selon les termes de la Licence Creative Commons Attribution - Pas d'Utilisation Commerciale 4.0 International. 


\title{
Japon-Corée, France-Algérie
}

\author{
Réflexions sur deux situations coloniales et postcoloniales \\ Japan-Korea, France-Algeria. Some Reflections on Two Colonial and Postcolonial \\ Situations
}

Lionel Babicz

1 Histoire, paix et réconciliation ${ }^{1}$. Si une quantité croissante de travaux sur ces thèmes ont été publiés en Asie orientale et en Europe, peu nombreuses sont les études comparatives. Le présent article résulte de ma participation à l'une de ces rares initiatives, un ouvrage japonais intituléHistoire et Réconciliation publié en 2011 par les historiens Kurosawa Fumitaka 黒沢文貴 et Ian Nish ${ }^{2}$. Ce livre, composé à la fois de travaux historiques et de témoignages directs, comporte également une partie comparative dédiée à des thèmes rarement abordés jusqu'à présent : mise en perspective des processus de réconciliation sino-japonais et germanopolonais, ou nippo-coréen et anglo-irlandais. Ma contribution personnelle fut une tentative de comparaison entre deux couples coloniaux n'ayant encore jamais été systématiquement confrontés, Japon-Corée et France-Algérie. Les quelques réflexions qui suivent sur divers aspects des situations coloniales et postcoloniales francoalgériennes et nippo-coréennes constituent une version remaniée et actualisée de ce texte japonais ${ }^{3}$.

\section{Le Japon et l'Allemagne}

2 La mémoire de guerre du Japon est souvent comparée à celle de l'Allemagne. Alors que l'Allemagne aurait courageusement fait face à son histoire, le Japon est décrit comme ayant tenté d'échapper à une telle confrontation avec ses méfaits passés. L'Allemagne est admirée pour s'être sincèrement réconciliée avec ses anciennes victimes et pour avoir adopté une éducation pacifiste fondée sur de sincères remords ${ }^{4}$. Le Japon, par contre, est souvent présenté comme un État refusant de reconnaître ses crimes passés et n'hésitant pas à provoquer la colère de ses voisins par des gestes intempestifs, comme les hommages de politiciens au sanctuaire Yasukuni. Qui plus est, l'éducation 
pacifiste japonaise ne serait pas fondée sur de véritables remords, mais sur un sentiment d'auto-victimisation. La principale victime de la guerre serait le peuple japonais lui-même, qui a subi l'horreur absolue des bombardements atomiques.

Une telle comparaison entre le Japon et l'Allemagne est certainement légitime. Après tout, les deux pays étaient alliés durant la Seconde Guerre mondiale, et de nombreux parallèles existent dans leur situation avant et durant la guerre. En outre, les deux États furent semblablement occupés et démilitarisés au sortir du conflit, avant de connaître une rapide et impressionnante reprise économique.

Cependant, la comparaison Japon-Allemagne peut également être trompeuse. En effet, l'histoire du Japon moderne d'avant 1945 n'est pas seulement l'histoire de guerres successives, mais aussi celle d'un colonialisme. Si le Japon fut bien en guerre avec la Chine entre 1937 et 1945, ou avec les États-Unis entre 1941 et 1945, un tel état de belligérance n'a jamais existé, par exemple, avec la Corée. La Corée ne fut jamais en état de guerre avec le Japon. Entre 1910 et 1945, la péninsule coréenne était officiellement annexée au Japon, et constituait en fait une colonie japonaise depuis 1905. La population et les ressources de la péninsule furent mobilisées pour appuyer l'effort de guerre japonais, et une partie de la population coréenne alla même jusqu'à sympathiser avec le colonisateur, s'identifier avec les Japonais et combattre à leurs côtés.

Qui plus est, le colonialisme japonais en Corée était motivé par des idées similaires (mais non identiques) à la politique menée par les Français en Algérie. La Corée, tout comme l'Algérie, faisait partie intégrante du territoire national, et les deux colonies étaient destinées, à terme, à être totalement assimilées au sein de la métropole. La mainmise japonaise sur la Corée fut ainsi une entreprise coloniale, et c'est à travers un prisme de décolonisation qu'il convient également de considérer le processus de réconciliation nippo-coréen en cours depuis 1945. Ces quelques pages se proposent ainsi d'examiner les parallèles et différences entre les situations coloniales francoalgériennes et nippo-coréennes, mais également de comparer la manière dont cette histoire porte une ombre encore aujourd'hui sur les relations au sein des deux « couples ».

\section{Le centenaire de l'annexion de la Corée}

6 Ces dernières années auront été emblématiques: les relations nippo-coréennes et franco-algériennes évoluent toujours à l'ombre de l'histoire et de la mémoire.

7 L'année 2010 marqua ainsi le centenaire de l'annexion de la Corée par le Japon ${ }^{5}$. En apparence, les choses se sont plutôt bien déroulées. L'événement a donné lieu à une série de manifestations d'amitié et de rapprochement entre Tōkyō et Séoul. Le Premier ministre japonais de l'époque, Kan Naoto 菅直人 (né en 1946), a publié une déclaration d'excuses forte et claire : la Corée fut assujettie au contrôle japonais contre la volonté du peuple coréen, la domination japonaise a privé les Coréens de leur pays et de leur culture et porté profondément atteinte à leur dignité ${ }^{6}$. Des propos bien accueillis en Corée du Sud, aussi bien au niveau officiel que dans les médias, et qui s'inscrivent dans le cadre d'une réelle proximité entre les deux pays.

8 Toutefois, la portée de cette déclaration est toute relative. En effet, ce ne sont pas là les premières excuses japonaises adressées à la Corée, tant s'en faut. La liste - certains diront la litanie - de celles-ci est même fort longue ${ }^{7}$. Les déclarations de Kan Naoto à 
l'occasion du centenaire de l'annexion ne constituent donc que le maillon supplémentaire d'une longue chaîne d'excuses, laquelle donne parfois au public japonais l'impression que la politique coréenne (et asiatique) de son pays se résume à une « diplomatie des excuses» (shazai gaikō 謝罪外交).

En Corée, même si officiellement on se félicite des récentes déclarations japonaises, on voit les choses différemment. Cette avalanche de paroles de contrition ne serait qu'un nuage de fumée destiné à masquer l'essentiel. Et l'essentiel, c'est le Tennō, le souverain du Japon (traduit dans les langues européennes par " empereur ", mais qui en coréen se traduit par « roi du Japon»), signataire du traité d'annexion de 1910. Certes, l'actuel Tennō, Akihito 明仁, s'est lui aussi excusé à maintes reprises pour les souffrances infligées à la Corée et à son peuple, mais il n'a jamais fait la moindre allusion au traité d'annexion lui-même ni à son prédécesseur au nom duquel l'annexion a été accomplie, son grand-père l'empereur Meiji.

En outre, malgré les invitations répétées du gouvernement coréen, Akihito ne s'est jamais rendu en Corée, alors qu'il a visité la Chine en 1992. En 2009, à la veille du centenaire de l'annexion, le président sud-coréen Yi Myŏngbak 李明博 (Lee Myungbak, né en 1941) avait convié l'empereur à marquer l'événement en effectuant une visite officielle dans la péninsule. Invitation déclinée par le gouvernement japonais, sans doute par crainte d'avoir à formuler des excuses d'une autre dimension, ayant trait à la légalité même de l'annexion.

11 En effet, depuis la normalisation de 1965 avec la République de Corée (Sud), la question de la légalité du traité d'annexion obscurcit les relations nippo-coréennes. Les conventions et traités ayant conduit à l'annexion de 1910 furent alors déclarés « déjà nuls et non avenus»(mohaya mukō de aru もはや無効である), formule ambiguë contournant la question de leur statut légal. Car pour Tōkyō - et ceci est toujours la position japonaise officielle - ces accords constituent certes une erreur historique pour laquelle il convient de faire amende honorable, mais leur légalité ne peut être mise en doute.

Ce n'est donc pas un hasard si, au lendemain de la déclaration de Kan Naoto, les médias coréens se faisaient largement l'écho de la découverte de différences entre les exemplaires coréen et japonais du traité d'annexion: la version coréenne ne comporterait ni la signature manuscrite de l'empereur Sunjong 純宗 (le roi de Corée avait pris le titre d'« empereur » en 1897) ni le sceau officiel requis. Ceci constituerait la preuve définitive de l'illégalité d'un traité scélérat imposé à la Corée par la force ${ }^{8}$.

Ainsi, la réelle proximité entre la Corée du Sud et le Japon masque des plaies encore à vif. Le centenaire de l'annexion aura permis de franchir un pas supplémentaire vers la réconciliation. Mais en déclinant l'invitation adressée par Séoul à l'empereur, le gouvernement japonais a manqué l'occasion d'accomplir le geste qui aurait pu refermer le douloureux chapitre de l'annexion et de la colonisation.

\section{Le cinquantenaire de l'indépendance de l'Algérie}

Pour ce qui est de la France et de l'Algérie, l'année 2012 aura marqué le cinquantième anniversaire de la fin de la guerre d'Algérie et de l'indépendance algérienne. Là aussi, polémiques et divergences furent au rendez-vous. Ainsi cet affrontement francofrançais autour des commémorations du 19 mars, date anniversaire du cessez-le-feu, à 
laquelle la Fédération nationale des anciens combattants en Afrique du Nord (Fnaca) honore depuis 1963 le souvenir des soldats tombés pour la France en Afrique du Nord. La date est attaquée par d'autres associations, qui n'y voient pas «l'anniversaire de la fin de la guerre, mais le début des massacres d'Européens et de harkis »'.

La reconnaissance officielle, par le nouveau président socialiste François Hollande, du massacre du 17 octobre 1961, a également suscité une vague de critiques ${ }^{10}$. François Hollande qui, à l'heure où sont rédigées ces lignes (octobre 2012), doit effectuer, en décembre 2012, une visite officielle en Algérie.

Au-delà des gestes politiques, il semble également que l'année 2012 aura été l'occasion pour la France intellectuelle, artistique et médiatique de se pencher sur le passé algérien comme cela n'avait jamais été fait auparavant. La quantité de publications, de manifestations, de diffusions consacrées à la guerre d'Algérie et, dans une moindre mesure, à la colonisation et à l'époque coloniale est impressionnante ${ }^{11}$. Certains thèmes sensibles, tel celui de la torture, ont également fait l'objet d'un traitement détaillé1 ${ }^{12}$. Ceci fait suite au grand nombre d'événements ayant marqué, quelques mois auparavant, le cinquantième anniversaire des événements du 17 octobre 1961 (du film de Yasmina Adi Ici on noie les Algériens à l'Appel pour la reconnaissance officielle de la tragédie du 17 octobre 1961 à Paris) ${ }^{13}$.

De manière apparemment paradoxale, le cinquantième anniversaire de l'indépendance algérienne semble avoir suscité plus d'intérêt en France qu'en Algérie, et ce malgré une série de célébrations officielles démarrant en juillet 2012. «Certes, écrit le journaliste Akram Belkaïd, quelques manifestations sont bien prévues çà et là. Bien sûr, le sujet est abordé par la presse, notamment par le biais d'articles mémoriels ou de biographies de militants disparus. Mais l'on ne sent pas la même frénésie, le même appétit à la fois culturel et artistique pour ce moment clé de l'histoire des Algériens » ${ }^{14}$.

En fait, la manière dont le Japon et la France ont respectivement célébré les anniversaires de 2010 et 2012 révèle, me semble-t-il, une différence essentielle entre les mémoires coloniales des deux pays. En France, l'Algérie suscite un important débat intérieur, passionnant une grande partie de la société civile. Au Japon, le passé colonial n'intéresse pas le grand public, et la colonisation de la Corée constitue aujourd'hui essentiellement une question diplomatique, une composante essentielle de l'un des liens stratégiques les plus importants du Japon contemporain - la relation avec la Corée du Sud -, et la société, mis à part quelques franges marginales, abandonne la gestion du passé aux dirigeants politiques.

\section{Hors-la-loi}

Mais qu'on ne s'y trompe pas. Entre la France et l'Algérie, les enjeux diplomatiques sont également fort importants et, là aussi, histoire et mémoire pèsent de tout leur poids. Le rapport au passé colonial n'est pas uniquement une affaire franco-française, mais également un élément fondamental de la relation franco-algérienne. Les débats suscités en 2010 par le film du réalisateur français d'origine algérienne Rachid Bouchareb, Horsla-loi, constituent une illustration de cette dimension multiple, interne et externe, de l'histoire du colonialisme français en Algérie ${ }^{15}$.

20 Hors-la-loi a pour thème les massacres de Sétif et Guelma - la répression sanglante par les Français de manifestations nationalistes survenues en Algérie en mai $1945^{16}$. Le 
8 mai, date du début de la crise, est devenu une fête nationale en Algérie. Cette date constitue un moment fondateur, et Sétif demeure dans la mémoire collective comme une grande blessure ${ }^{17}$.

21 Il aura suffi de l'annonce de la sortie du film pour que resurgissent immédiatement «les guerres de mémoire " ${ }^{18}$. D'un côté, les partisans du film, au premier rang desquels la presse algérienne, comme le quotidien El Watan qui parle de " film qui dérange $»^{19}$. En face, les associations de Français rapatriés d'Algérie dénonçant "un film mensonger » qui donnerait «la version du FLN» de l'histoire algérienne «tout en prétendant à la vérité historique $»^{20}$.

L'affaire Hors-la-loi est emblématique de la sensibilité de la question algérienne dans la France d'aujourd'hui, de l'état des relations entre les deux pays, et du poids de l'histoire sur ces rapports. Tout comme dans le cas du Japon et de la Corée du Sud, la France et l'Algérie n'ont pas encore refermé le chapitre postcolonial de leur histoire commune. "Les relations entre la France et l'Algérie peuvent être bonnes ou mauvaises, en aucun cas elles ne peuvent être banales $»^{21}$. Cette fameuse phrase du président algérien Abdelaziz Bouteflika (né en 1937), prononcée en 1974, décrit toujours l'état des relations entre les deux pays. Le spécialiste de l'Algérie Jean-François Daguzan décrit ainsi une " perpétuelle leçon de tango » : un pas en avant, deux pas en arrière ${ }^{22}$. Quant au journaliste du Monde Jean-Pierre Tuquoi, il parle de « couple infernal ${ }^{23}$. Des clichés rappelant les expressions ressassées utilisées pour décrire les relations nippocoréennes, comme la formule des "deux pays si proches, et pourtant si éloignés » (chikakute töi kuni 近くて遠い国).

\section{La force du lien colonial}

Aussi bien dans le cas de la France et de l'Algérie que dans celui du Japon et de la Corée, on ne peut véritablement saisir l'état des relations bilatérales sans se référer à la réalité de la période coloniale. Quelles sont donc les similitudes et les différences dans les situations coloniales franco-algérienne et nippo-coréenne?

La similarité la plus frappante est peut-être la spécificité de la relation, la force du lien qui reliait les deux partenaires coloniaux.

L'Algérie fut une colonie française durant plus de cent trente ans (1830-1962); colonisation partielle et progressive avant 1871, puis totale ensuite. En outre, à la différence du Maroc et de la Tunisie conquis au $\mathrm{xx}^{\mathrm{e}}$ siècle et définis comme protectorats, l'Algérie était une colonie de peuplement, constituée de trois départements administrativement rattachés à la métropole. L'Algérie était donc la possession française la plus ancienne et la plus importante, indispensable stratégiquement (l'Algérie était la clé de l'Afrique, du Maghreb et du Proche-Orient), et aussi symbole de la grandeur de la France ${ }^{24}$.

"L'Algérie, c'est la France! », déclarait en novembre 1954, lors du déclenchement de l'insurrection algérienne, le ministre de l'Intérieur François Mitterrand ${ }^{25}$. L'Algérie n'était en effet ni une colonie lointaine, comme le Sénégal ou le Gabon, ni un protectorat comme le Maroc ou la Tunisie. Un million d'Européens, les «pieds-noirs ", y vivaient depuis des générations. D'ailleurs, l'Algérie ne relevait pas du ministère des Colonies, mais du ministère de l'Intérieur - comme tout autre département français ${ }^{26}$. 

comparable avec la mainmise japonaise sur la Corée. Pendant quarante ans, de 1830 à 1871, l'Algérie fut gouvernée par l'armée. Durant ces années, l'histoire de l'Algérie fut largement celle de l'insurrection des tribus et des sociétés villageoises ${ }^{27}$. La défaite française de 1870 face à la Prusse et la fin du Second Empire (1852-1870) modifièrent la donne. À partir de 1871, l'Algérie passa aux mains de l'administration civile, et se vit définie comme le prolongement de la France de l'autre côté de la Méditerranée, un immense territoire destiné à être « francisé » et assimilé à la France ${ }^{28}$.

La Corée, elle aussi, constituait une partie inaliénable du territoire japonais ${ }^{29}$. La péninsule avait été «rattachée, unifiée » (heigō 併合) au Japon. La Corée n'était pas définie comme " colonie » (shokuminchi 植民地), mais était une " province extérieure " (gaichi 外地), appelée la plupart du temps simplement «la péninsule» (hantō 半島). Peuplée de colons japonais - comme l'Algérie l'était de colons français - la Corée avait le statut de conquête majeure du Japon moderne. C'était un territoire avec lequel non seulement les liens étaient extrêmement anciens, mais dont l'importance stratégique était vitale. Le Japon avait, depuis le début de l'ère Meiji, considéré la stabilité de la Corée comme un élément fondamental de sa sécurité nationale. La Corée avait constitué l'enjeu essentiel des guerres sino-japonaise (1894-1895) et russo-japonaise (1904-1905), et le protectorat de 1905 puis l'annexion de 1910 étaient en grande partie l'aboutissement de cette fixation du Japon moderne sur la question coréenne.

Le Japon se voyait également porteur d'une mission civilisatrice à l'égard de la Corée, tout comme la France en Algérie. Napoléon III aspirait à sauvegarder la société arabe en l'amenant progressivement vers la modernité ${ }^{30}$. Le Japon désirait guider la péninsule le long du chemin que lui-même avait suivi avec succès depuis la restauration de Meiji.

\section{Deux catégories de personnes}

Il y avait une différence, cependant. Le Japon avait développé à l'égard de la Corée une idéologie de proximité raciale. Les Coréens étaient considérés comme des frères de race égarés, destinés au bout du compte à regagner le bercail originel d'une civilisation commune. Ce sont ces idées, déjà présentes au moment de l'annexion en 1910, qui furent à la base des mesures d'assimilation (kōminka seisaku 皇民化政策) mises en place à partir de 1938.

En Algérie, pas de proximité raciale qui tienne. L'Algérie était destinée à être assimilée à la France par le biais d'une colonisation française (et européenne) et par la «francisation " progressive d'une élite locale. Durant toute l'époque coloniale, il y eut essentiellement deux catégories de personne en Algérie : les colons - citoyens français bénéficiant de tous les droits - (auxquels furent adjoints en 1870 les Juifs algériens), et les indigènes locaux, les "Arabes ", régis par le Code de l'indigénat de $1881^{31}$. Ainsi, lorsque l'on parle d'assimilation, il s'agit de l'assimilation des citoyens colons, et non de celle des Algériens musulmans.

En Corée coloniale, la situation était infiniment plus complexe. Le statut légal de la Corée (et des autres colonies japonaises) était en constante évolution, car considéré comme provisoire. L'objectif final était une totale intégration au système légal japonais, mais la réalité exigeait que chaque colonie développe son propre système juridique reflétant les conditions locales ${ }^{32}$. La population coréenne était soumise pour sa part aux 
décrets du gouverneur général (lequel dépendait directement de l'empereur) et partant de cela, à une profonde discrimination. Ainsi, dans les deux colonies, au nom d'une lointaine et utopique assimilation, les populations indigènes étaient l'objet d'une ségrégation intens ${ }^{33}$, et les opposants les plus farouches à l'assimilation des Coréens étaient en fait les colons japonais, craignant de perdre leurs privilèges ${ }^{34}$.

À noter toutefois, dans le cas du Japon, une égalité théorique pour les Coréens résidant en métropole. Entre 1925 et 1945, les hommes coréens âgés de plus de vingt-cinq ans qui demeuraient au Japon, du fait de leur lieu de résidence, possédaient le droit de vote ainsi que celui d'être candidat aux élections législatives et municipales. Parallèlement, les Japonais installés en Corée étaient privés de ces droits. Cette situation entrouvrit les portes de la politique japonaise, à partir de 1932, à un petit nombre de Coréens, le plus célèbre d'entre eux étant l'entrepreneur Pak Ch'ungŭm 朴春琴 (1891-1973) ${ }^{35}$.

Le statut de la Corée fut aménagé durant la guerre du Pacifique. Y furent appliquées la Constitution de Meiji (à l'exception des droits politiques propres aux sujets de l'empereur) ainsi qu'un important nombre de lois japonaises. Le gouverneur perdit nominalement son autonomie, et le territoire passa sous l'autorité du ministère de l'Intérieur. D'autre part, pour la première fois, des députés coréens issus de Corée devaient maintenant être élus à la Diète à Tōkyō, et de nouvelles voies d'ascension sociale, notamment au sein de la hiérarchie militaire, s'ouvrirent. Ces premiers pas furent interrompus par la défaite de 1945. Il est intéressant de noter que ces mesures allaient toutes dans le sens de l'intégration.

Le statut de l'Algérie, lui, ne sera aménagé qu'en 1947, après la Seconde Guerre mondiale, et en réaction aux émeutes de 1945, d'où un aménagement fort différent de celui effectué en Corée par les Japonais durant la guerre. Si le Japon avait toujours évité d'établir des assemblées coloniales, craignant qu'elles ne conduisent à plus d'autonomie et à moins d'intégration, la France, pour sa part, établit en 1947 une "Assemblée algérienne». Celle-ci devait être formée de deux collèges paritaires de soixante délégués chacun, l'un représentant les citoyens français et une minorité de musulmans, l'autre le million et demi de musulmans ayant le droit de vote. Malgré ces conditions discriminatoires, les Algériens répondirent avec enthousiasme aux nouvelles possibilités qui s'ouvraient à eux. Craignant une victoire nationaliste, les autorités coloniales choisirent de truquer les élections de $1948^{36}$.

36 À la même époque, la Corée, libérée du colonialisme japonais, se retrouvait aux avantpostes de la guerre froide. En 1948, le pays était divisé, avant de connaître une guerre d'une violence sans commune mesure avec celle de la période coloniale japonaise - la guerre de Corée (1950-1953).

\section{Décalage chronologique, décolonisation}

Ce décalage des chronologies constitue peut-être l'une des différences essentielles entre les deux situations coloniales. L'Algérie est colonisée beaucoup plus tôt que la Corée (prise d'Alger en 1830, colonisation officielle à partir de 1905 pour la péninsule), et se libère plus tard (la Corée est libérée en 1945, l'Algérie se libère en 1962) ${ }^{37}$. Ainsi, l'étreinte franco-algérienne fut bien plus étroite que l'embrassement nippo-coréen. Des générations de colons se succédèrent en Algérie, alors que beaucoup de colons japonais 
connurent, au cours de leur propre vie, à la fois la conquête et le rapatriement lié à la défaite contre les alliés. million et demi de soldats français ont combattu en Algérie, et à leurs côtés quelque cent mille harkis. À la fin de la guerre, un million de pieds-noirs ont regagné la France métropolitaine. Ainsi, aujourd'hui en France, quatre à cinq millions de personnes - des pieds-noirs, des harkis, des soldats, des immigrés algériens - sont encore directement concernées par la guerre d'Algérie ${ }^{41}$. Tout ceci explique, entre autres, pourquoi «tout entre les deux pays est passionné et dramatique ${ }^{42}$.

\section{Occultation}

Or, malgré l'ampleur de ces événements, le terme de "guerre » ne fut officiellement employé en France pour la première fois qu'en $1999^{43}$, lorsqu'une loi substitua aux termes "opérations effectuées en Afrique du Nord » l'expression "guerre d'Algérie " ou « combats en Tunisie et au Maroc $»^{44}$. Jusqu'alors, on parlait de maintien de l'ordre, de pacification, de rebelles, d'événements ${ }^{45}$. en 1991, un ouvrage intitulé La gangrène et l'oubli, dans lequel il analysait comment cette « guerre sans nom » demeurait une page blanche de l'histoire de France, et « rongeait comme une gangrène les fondements mêmes de la société ${ }^{46}$.

Occultation également en Algérie, mais d'un autre ordre. La guerre y était célébrée représentait un mythe fondateur, mais la réalité des événements était modifiée. La guerre contre la France était une "révolution », et la guerre civile qui opposa le Front de libération nationale (FLN) et le Mouvement national algérien (MNA) était passée sous silence, tout comme les massacres de harkis ${ }^{47}$. 

négociations en vue d'une normalisation des relations, qui s'ouvrirent en 1951 , n'aboutirent que quatorze ans plus tard, en 1965. Le Japon avait éliminé la Corée de sa conscience, et s'était replié sur lui-même pour soigner ses blessures et se régénérer. Ainsi, même si les raisons en étaient différentes, un phénomène similaire d'occultation du passé se produisit en France et au Japon au sortir de la période coloniale.

\section{Normalisation nippo-coréenne}

En 1965, donc, le Japon établissait des relations diplomatiques avec la République de Corée, au Sud. Ce traité de normalisation était le résultat à la fois de pressions des États-Unis, qui aspiraient à renforcer la stabilité régionale, et d'une convergence d'intérêts, essentiellement économiques, entre la Corée et le Japon. Les investisseurs japonais étaient attirés par le marché coréen, et la Corée avide de capitaux japonais. Pour le régime militaire de Pak Chŏnghŭi 朴正熙 (Park Chung Hee, 1917-1979), l'accord avec le Japon s'inscrivait aussi dans le contexte d'un resserrement des liens avec les États-Unis - en 1965 la Corée envoyait des troupes au Vietnam - et d'une méfiance accrue à l'égard de la République populaire de Chine, qui avait atteint l'année précédente le statut de puissance nucléaire. 

du traité consistait en l'établissement de relations diplomatiques et en l'octroi, par le Japon, d'une importante aide économique à la Corée du Sud. Le reste n'était que désaccords et ambivalences.

Une première ambivalence avait trait au statut du gouvernement sud-coréen. Celui-ci était-il le gouvernement légal de l'ensemble de la Corée (selon l'interprétation de Séoul), ou bien seulement du sud de la péninsule (selon l'interprétation de Tōkyō, qui désirait laisser une porte entrouverte en direction de la Corée du Nord)?

ingivalence la plus importante avait trait à l'histoire. L'ensemble des accords et traités conclus avant le 22 août 1910 entre le Japon et la Corée étaient donc déclarés comme "déjà nuls et non avenus", une formulation qui évitait de déterminer clairement le statut du traité de protectorat de 1905 et du traité d'annexion de 1910. Selon la position officielle japonaise, ces accords avaient été conclus légalement, et la validité du traité d'annexion avait pris fin le 15 août 1948, avec la création de la Corée du Sud. Pour Séoul, il s'agissait de documents signés sous la contrainte et dépourvus de toute légitimité ou validité.

La formule était à l'avantage du Japon, qui lui permit de ne pas inclure d'excuses formelles pour la colonisation dans le traité ni de verser des indemnités pour la période coloniale. Cependant, lorsqu'il arriva à Séoul pour signer le traité, le ministre des Affaires étrangères japonais, Shiina Etsusaburō 椎名悦二郎 (1897-1979), exprima les regrets de son pays pour ce "passé infortuné ». Et Tōkyō octroya à Séoul une aide économique massive, sous forme de dons et de prêts. Toutefois, officiellement, ceci n'avait aucun lien avec le passé. Malgré ses imperfections, le traité de 1965 marqua une étape importante. Il permit d'ouvrir un nouveau chapitre, et contribua grandement au démarrage économique sud-coréen.

\section{La France et l'Algérie après l'indépendance}

Contrairement au cas nippo-coréen où l'indépendance amena une rupture des liens bilatéraux, entre la France et l'Algérie le contact ne fut jamais rompu. Il est même surprenant de voir comment, malgré la violence et les atrocités de la guerre, dès son lendemain, les deux parties manifestèrent leur volonté de maintenir un lien étroit ${ }^{49}$.

Ce qui ne veut pas dire que les rapports franco-algériens furent idylliques, tant s'en faut. La politique française à l'égard de l'Algérie au lendemain de l'indépendance fut définie comme une politique de " coopération ", à savoir l'octroi d'une aide massive de l'ancienne puissance coloniale à l'État algérien indépendant. Jusque dans les années 1970, l'Algérie va ainsi représenter le plus gros poste budgétaire de la coopération française. Cette aide massive se poursuivit malgré des crises répétées autour des diverses nationalisations, notamment celle des hydrocarbures, mises en œuvre par le gouvernement algérien et auxquelles s'opposait vigoureusement la France.

En 1981, le président François Mitterrand se rendit même à Alger pour une visite d'une grande portée symbolique. Accès aux archives de l'Algérie française, signature d'un accord gazier (la France acceptait d'acquérir le gaz algérien plus cher que le prix du marché) : là encore, les gestes de bonne volonté se succédèrent. 


\section{Dégradation franco-algérienne, amélioration nippo- coréenne}

Mais l'année 1981 marqua également un pic. Depuis, à l'exception de quelques embellies temporaires, les relations ont été se dégradant. Au départ, la principale raison de cette détérioration fut économique. Dans les années 1980, la France, confrontée une situation économique mondiale de plus en plus difficile, ne pouvait plus continuer à payer le gaz algérien au prix fort et demandait une révision des accords conclus à ce sujet. Autre sujet de tension : après la vague d'attentats de 1986, la France décida de rétablir les visas d'entrée pour les non-résidents de la Communauté européenne. Cette mesure fut ressentie comme une offense au Maghreb et particulièrement en Algérie, qui établit rapidement la réciprocité ${ }^{50}$.

Entre le Japon et la Corée du Sud, également, tensions et crises n'ont pas manqué. L'un des incidents les plus graves eut lieu en 1973, autour de l'enlèvement, par les services de sécurité sud-coréens, de l'opposant Kim Daejung 金大中 (1925-2009) ${ }^{51}$ alors à Tōkyō en fuite depuis le régime militaire de Séoul. Cette affaire propulsa la Corée à la Une des médias japonais. Protestations contre la violation de la souveraineté nationale et dénonciation $d u$ caractère dictatorial du régime sud-coréen : après une éclipse d'une trentaine d'années, voilà que la Corée regagnait l'ordre du jour public japonais. Les dramatiques événements des années qui suivirent - en 1979, l'assassinat du président Pak Chŏnhŭi et le coup d'État qui porta au pouvoir son ex-bras droit Chŏn Tuhwan 全斗 煥 (Chun Doo Hwan, né en 1931), en 1980, le massacre de Kwangju et la condamnation à mort contre Kim Daejung ${ }^{52}$ - devaient contribuer au maintien de cet intérêt, mais également renforcer l'image négative du pays voisin, ressenti comme brutal, de fait une dictature militaire.

Le véritable tournant dans les relations se produisit avec l'accession au poste de Premier ministre de Nakasone Yasuhiro 中兽根康弘 (né en 1918) en 1982. Le Premier ministre Nakasone était persuadé de la nécessité stratégique, économique et politique, d'un rapprochement avec la Corée du Sud. Il effectua son premier déplacement à l'étranger, en 1983, en Corée du Sud. Ce voyage constituait la première visite officielle d'un chef de gouvernement japonais en Corée. Nakasone promit à Séoul une importante assistance financière, et les dirigeants des deux pays proclamèrent l'ouverture d'un «nouveau partenariat nippo-coréen » (nijū isseki ni muketa arata na nikkan pātonāshippu 21世紀に向けた新たな日韓パートナーシップ $)^{53}$. Quelques mois plus tard, le président Chŏn Tuhwan se rendait au Japon, afin d'y sceller une alliance conservatrice américano-nippo-coréenne.

Ce rapprochement était d'autant plus remarquable qu'il intervenait au lendemain de la première crise des manuels scolaires de 1982. Cette crise fut résolue au moyen d'une directive gouvernementale demandant aux enseignants de tenir compte des critiques émises par les États de la région, et d'une promesse de futures révisions. Effectivement, dans les années qui suivirent, un certain nombre de changements sémantiques furent introduits dans les ouvrages incriminés, et le différend s'apaisa, du moins au niveau officiel. 


\section{Un Mondial à l'ombre du passé}

61 Une étape supplémentaire importante du processus de réconciliation fut franchie en 1998, lors d'une rencontre au sommet entre le président coréen Kim Daejung et le Premier ministre japonais Obuchi Keizō 小㴊恵三 (1937-2000).

Cette rencontre constituait la tentative la plus spectaculaire pour clore le chapitre de la colonisation. C'était la première fois que les dirigeants des deux États faisaient référence au passé dans un document écrit officiel. À la veille du xxi siècle, le Japon et la Corée du Sud décidaient de tourner leurs regards vers l'avenir, et accompagnaient cette résolution d'une série d'accords sans précédent.

63 L'une des causes de ce spectaculaire rapprochement était la proximité du Mondial de 2002. La Fédération internationale de football, ne pouvant départager le Japon et la Corée du Sud, avait invité les deux pays, en 1996, à organiser conjointement la compétition. Cette décision sans précédent avait été imposée par la douloureuse histoire des relations nippo-coréennes. Le choix de l'un aurait atteint l'autre au plus profond de sa dignité nationale.

64 Cette collaboration forcée fut acceptée avec réluctance par les deux parties. Mais le choc initial passé, on fut bien obligé de faire contre mauvaise fortune bon cœur. L'arrivée au pouvoir de Kim Daejung, en 1997, devait également faciliter les choses. L'ancien opposant était mieux disposé à l'égard du Japon que ses prédécesseurs, et était persuadé de la nécessité de tourner la page sur le passé et de regarder vers l'avenir.

65 Mais les difficultés devaient encore être importantes. En premier lieu, l'organisation même du Mondial donna lieu à d'interminables désaccords. Depuis l'appellation officielle de la compétition (Japon-Corée ou Corée-Japon - finalement, les deux appellations furent adoptées, la première au Japon et la seconde en Corée du Sud) jusqu'à la question du lieu de la cérémonie d'ouverture (la Corée fut choisie) et de clôture (le Japon). Mais l'événement qui devait mettre en péril la tenue même du Mondial fut la seconde crise des manuels d'histoire, qui éclata en 2001 autour du Nouveau manuel d'histoire (Atarashii rekishi kyōkasho 新しい歴史教科書) de l'organisation Tsukuru-kai (Atarashii rekishi kyōkasho o tsukuru-kai 新しい歴史教科書をつくる 会 $)^{54}$.

66 Cependant, à la veille de l'ouverture des compétitions, les gestes de bonne volonté se succédèrent. L'empereur alla ainsi jusqu'à rappeler l'ascendance coréenne de la mère d'un de ses lointains prédécesseurs, Kanmu 桓武天皇 (780-806). Le monarque japonais renonça, cependant, à se rendre en Corée pour les cérémonies d'ouverture, comme cela avait été un temps envisagé. Ainsi fut manquée l'occasion d'une visite historique, qui aurait contribué, accompagnée des gestes et déclaration appropriés, à clore le chapitre de l'après-guerre et de l'époque postcoloniale ${ }^{55}$.

Malgré ce ratage, le Mondial nippo-coréen de 2002 fut une incontestable réussite. C'était la première fois que les deux pays organisaient conjointement un événement d'une telle ampleur. Mieux encore : l'année 2002 vit le début, au Japon, d'un boom culturel coréen d'une ampleur sans précédent. La Corée du Sud devint subitement un pays qui attisait la curiosité d'un large public. Le phénomène n'a fait que s'amplifier depuis, et est tangible dans les domaines les plus divers. 


\section{Vers la fin de l'époque postcoloniale?}

Aujourd'hui, plus d'un siècle après l'annexion, plus de soixante-cinq ans après l'indépendance, les signes annonciateurs de la fin de l'époque postcoloniale nippocoréenne vont se multipliant. Néanmoins, celle-ci refuse aussi obstinément de s'achever. La cause principale de cette persistance est le poids de l'histoire. L'incapacité de l'accord de normalisation de 1965 à affronter les questions historiques se fait ainsi sentir jusqu'à aujourd'hui. La légitimité et la validité du traité d'annexion font toujours l'objet d'un vif débat. Et la controverse autour de la souveraineté sur les rochers Liancourt (en japonais Takeshima 竹島, en Coréen Tokto 獨島) se poursuit.

Néanmoins, le centenaire, malgré les ratages évoqués plus haut, aura également permis de mesurer l'immensité du chemin parcouru sur la voie de la réconciliation. En dépit du passé colonial, le Japon et la Corée du Sud sont aujourd'hui des partenaires privilégiés. Les traités de sécurité liant Séoul et Tōkyō à Washington ont l'allure d'une véritable alliance trilatérale, et il ne fait guère de doute que ce triangle de sécurité est à la base de la paix dont jouit l'Asie orientale depuis un demi-siècle ${ }^{56}$.

\section{L'Algérie et la France dans la tourmente}

70 En Algérie, par contre, depuis 1988, c'est la tourmente, et les événements dramatiques que traverse le pays, couplés à une menace islamiste globale, affectent fortement les relations avec la France.

Un bref rappel : en 1988, l'armée algérienne ouvre le feu sur des manifestants, causant la mort de plus de cinq cents personnes. En 1991, le Front islamique du salut (FIS) remporte le premier tour des élections législatives. L'armée s'empare alors du pouvoir et annule les élections. En 1992, l'Algérie entre en guerre civile, une guerre qui atteint également la France en 1994 avec une série d'attentats. En Algérie même, le bilan de cette guerre est imposant et atteint plus de cent cinquante mille morts à ce jour. Le GIA (Groupe islamique armé) a été défait par le gouvernement et, depuis 1999, la violence a notablement diminué. Cependant, le gouvernement doit aujourd'hui faire face à un nouvel adversaire : Al-Qaida au Maghreb islamique (AQMI), qui menace également la France. La violence est toujours endémique, et une vingtaine de personnes sont tuées en moyenne chaque mois en Algérie.

2 Dans les années 1990, la France, touchée alors par la violence algérienne, balança entre une défense stricte des droits de l'homme (face aux détentions arbitraires et disparitions dues au pouvoir algérien) et la lutte contre le terrorisme. Ce débat sur le "Qui tue qui ?» ombragea alors fortement les relations bilatérales, toute remarque française étant ressentie par le gouvernement algérien comme une ingérence dans les affaires intérieures du pays ${ }^{57}$.

3 À partir de 1999, au sortir de la première phase de la guerre civile, la France s'efforça de reconstruire sa relation avec l'Algérie sur des bases symboliques. Le président Jacques Chirac essaya notamment, mais en vain de parvenir à la signature d'un "traité d'amitié » entre les deux pays et, en 2003, il se rendit en visite officielle en Algérie.

Mais depuis 2005, les relations entre les deux pays se sont à nouveau dégradées, et ce particulièrement autour du thème de l'histoire et de la mémoire. Le président Bouteflika s'en est ainsi pris à la France, assimilant les « crimes » de celle-ci aux crimes 
nazis (utilisation de "fours" à chaux pour brûler les cadavres, accusation de « génocide » larvé) $)^{58}$.

\section{Repentance}

Le président Nicolas Sarkozy, tout en rejetant toute idée de repentance, reconnut, en 2007, sur le sol algérien, la profonde injustice du système colonial :

Oui, le système colonial a été profondément injuste, contraire aux trois mots fondateurs de notre République : liberté, égalité, fraternité. Mais il est aussi juste de dire qu'à l'intérieur de ce système, profondément injuste, il y avait beaucoup d'hommes et de femmes qui ont aimé l'Algérie, avant de devoir la quitter. Oui, des crimes terribles ont été commis tout au long d'une guerre d'indépendance qui a fait d'innombrables victimes des deux côtés. Et aujourd'hui, moi qui avais sept ans en 1962, c'est toutes les victimes que je veux honorer ${ }^{59}$.

Cependant, la France officielle semble de plus en plus disposée à effectuer des actes de repentance, particulièrement au sujet d'épisodes précis. Ainsi Sétif et Guelma : en 2005, déjà, l'ambassadeur de France en Algérie, Hubert Colin de Verdière, tenait des propos sans précédent à l'Université de Sétif: "Fallait-il, hélas, qu'il y ait sur cette terre un abîme d'incompréhension entre les communautés, pour que se produise cet enchaînement d'un climat de peur, de manifestations et de leur répression, d'assassinats et de massacres !", déclarait-il ${ }^{60}$. En 2008, l'ambassadeur Bernard Bajolet, toujours à Sétif, évoquait à son tour "d'épouvantables massacres [...] qui ont fait insulte aux principes fondateurs de la République française et marqué son histoire d'une tache indélébile ${ }^{61}$. Ainsi, plus récemment, la reconnaissance du massacre du 17 octobre 1961 par François Hollande ${ }^{62}$.

\section{Éloge de la colonisation}

Mais ces gestes ne constituent qu'un pan du tableau. En effet, on assiste parallèlement à un retour de l'éloge de la colonisation. Ainsi, en 2005, était adoptée par le Parlement une loi « portant reconnaissance de la Nation et contribution nationale en faveur des Français rapatriés ", et demandant que "les programmes scolaires reconnaissent en particulier le rôle positif de la présence française outre-mer, notamment en Afrique du Nord $»^{63}$.

Ce texte n'avait pas pour objet spécifique l'Algérie, mais il suscita de vives réactions, à la fois en France, où une pétition d'historiens reçut plus d'un millier de signatures ${ }^{64}$, et dans les anciennes colonies, et particulièrement en Algérie, où le président Bouteflika parla de « cécité mentale, confinant au négationnisme et au révisionnisme » ${ }^{65}$.

79 En 2006, le $2^{\mathrm{e}}$ alinéa de l'article 4 (sur « le rôle positif de la présence française outremer ») fut abrogé par le Conseil constitutionnel, sans débat parlementaire, entraînant la protestation de députés de droite ${ }^{66}$. Parallèlement, il était décidé de ne pas mettre en œuvre l'article 3 de la loi, prévoyant la création d'une « Fondation pour la mémoire de la guerre d'Algérie, des combats du Maroc et de Tunisie ». Décision renversée en 2010 avec l'établissement de ladite Fondation à l'Hôtel national des Invalides ${ }^{67}$. 


\section{Une surabondance mémorielle}

80 On le voit: si, au lendemain de la guerre d'Algérie, c'était silence et occultation qui dominaient ${ }^{68}$, aujourd'hui la situation s'est renversée, et l'on est passé à une "surabondance mémorielle", voire à une "guerre des mémoires " situation entre le Japon et la Corée du Sud. Certes, les deux pays entretiennent, à bien des égards, des relations plus proches que la France et l'Algérie, proximité due à la similarité des systèmes politiques, économiques et sociaux, à l'alliance militaire avec les États-Unis, à des craintes en partie partagées face à la Corée du Nord (et à la République populaire de Chine). Néanmoins, là aussi, l'histoire pèse de tout son poids, et la mémoire du passé ombrage le présent.

81 Tel est le cas, récemment, avec le conflit territorial autour des rochers Liancourt (Tokto/Takeshima), lui aussi fruit de l'histoire. Les rochers Liancourt - nommés par référence au baleinier français Le Liancourt, qui les découvrit en 1849 - sont un minuscule archipel constitué de deux îlots rocheux et d'une centaine de récifs, situé au large de la mer du Japon (ou mer Orientale pour les Coréens), à mi-chemin entre le Japon et la Corée. Les Japonais appellent ces îles Takeshima, et les Coréens Tokto.

Les rochers sont actuellement sous souveraineté coréenne, mais font l'objet d'une revendication japonaise s'appuyant sur le fait qu'ils ne sont pas mentionnés dans le traité de paix de San Francisco (1951). Le Japon affirme avoir contrôlé l'archipel durant des siècles, avant de l'incorporer au département de Shimane en 1905. Les Coréens rejettent cette argumentation, soutenant que leur pays a de tout temps gouverné ces îlots, et que l'incorporation au département de Shimane fut un acte illégitime accompli dans le contexte du colonialisme japonais de l'époque.

Cette question est ainsi intimement liée à la vision et à l'interprétation de l'histoire moderne des relations nippo-coréennes. En 1965, lorsque le Japon et la Corée du Sud normalisèrent leurs relations, la question fut éludée au moyen d'un échange de notes affirmant que tout conflit entre les deux pays serait résolu par des moyens diplomatiques. À ce jour, le différend territorial des rochers Liancourt est toujours pendant, et la question ne cesse de hanter les relations bilatérales.

Les couples franco-algérien et nippo-coréen semblent ainsi constituer deux variantes d'une même situation postcoloniale. Les deux couples entretiennent aujourd'hui des relations dictées essentiellement par leurs situations géostratégiques et leurs choix politiques. Dans ce contexte, la mémoire du passé colonial est souvent instrumentalisée pour des besoins intérieurs et extérieurs. Mais ces débats, disputes, accrochages et affrontements sont également l'expression de plaies toujours vives, et qui ne semblent pas près de se refermer. La colonisation travaille toujours en profondeur tant les sociétés des pays colonisateurs que celles des pays colonisés ${ }^{70}$. 


\section{NOTES}

1. La rédaction de cet article a bénéficié du soutien de l'Académie des Études coréennes (Academy of Korean Studies AKS-2010-R48) et de l'Université de Sydney (Faculty of Arts Research Support Scheme). Je remercie également Mary Nasr pour son assistance.

2. Kurosawa Fumitaka et Ian Nish (dir.), Rekishi to wakai 歴史と和解, Tōkyō daigaku shuppankai 東京大学出版界, 2011, 433 p.

3. M'adressant ici à un lectorat francophone, j'ai notamment raccourci certains développements sur l'Algérie et élargi d'autres parties consacrées à la Corée.

4. Lily Gardner Feldman, “The Role of History in Germany's Foreign Policy of Reconciliation: Principle and Practice", in Opening Historical Reconciliation in East Asia through Historical Dialogue (Proceedings of the First International Forum on Historical Reconciliation in East Asia), Seoul, Northeast Asian History Foundation, 2009, 290 p.

5. Les remarques suivantes sur le centenaire de l'annexion de la Corée ont déjà été en partie publiées sur le site internet du Monde, le 15 septembre 2010 ; Lionel Babicz, " Tōkyō-Séoul : une occasion manquée?»

6. «Kankoku heigō hyaku-nen: shushō danwa no zenbun» 韓国併合100年 首相談話の全文 (Centenaire de l'annexion de la Corée : texte intégral de la déclaration du Premier ministre), Asahi shinbun 朝日新聞, 10 août 2010.

7. Lionel Babicz, « Japon-Corée : de vaines excuses? », Raison publique, 10 mai 2009, p. 17-28.

8. Tae-hoon Lee, "1910 Japan-Korea annexation treaty invalid", The Korea Times, 11 août 2010. Voir également l'article d'Arnaud Nanta, dans le présent volume.

9. Propos tenus par Philippe Schmitt, président de l'Union nationale des combattants. Hélène Sallon, « Guerre d'Algérie, bataille mémorielle », Le Monde, 16 mars 2012.

10. "Hollande reconnaît la répression du 17 octobre 1961, critiques à droite", Le Monde, 17 octobre 2012 ; Paulette Péju, Gilles Manceron, Marcel Péju, Le 17 octobre des Algériens, suivi de La triple occultation d'un massacre, La Découverte, 2011, 199 p.

11. Un seul exemple : la série consacrée à l'Algérie par l'émission de France Inter La marche de l'histoire (12-16 mars 2012). Seule la première émission était consacrée au «temps colonial », les quatre suivantes traitant de la guerre d'Algérie.

12. Voir par exemple le visuel interactif "La torture et la guerre d'Algérie » mis en ligne le 17 mars 2012.

13. "Appel pour la reconnaissance officielle de la tragédie du 17 octobre 1961 à Paris ", Médiapart, 12 octobre 2011.

14. Akram Belkaïd, "Guerre d'Algérie : quand la France fait son travail de mémoire ", Slate Afrique, 10 mars 2012.

15. Rachid Bouchareb, «En France, “certains n'ont toujours pas accepté la décolonisation” », Le Monde, 21 mai 2010.

16. Le bilan officiel est de 102 morts européens et 1165 morts algériens. Les Algériens évoquent de 45000 à 80000 morts. Jean-Pierre Peyroulou, "Setif and Guelma (May 1945)", Online Encyclopedia of Mass Violence.

17. Benjamin Stora, "France-Algérie : "La simple reconnaissance des faits commis est très importante" ", Le Monde, 21 mai 2010.

18. Yasmina Adi, Didier Daeninckx et coll., «Le film "Hors-la-loi" de Rachid Bouchareb: les guerres de mémoires sont de retour », Le Monde, 5 mai 2010.

19. El Watan, 29 septembre 2010.

20. Abel Mestre et Caroline Monnot, «Qui est à l'origine des manifestations contre le film "Hors la loi" ?», Le Monde, 21 septembre 2010. 
21. Cité par Nicole Grimaud, La politique extérieure de l'Algérie: 1962-1978, Khartala, 2000, 366 p., p. 39.

22. Jean-François Daguzan, «Les relations franco-algériennes ou la perpétuelle leçon de tango », Maghreb Machrek, $n^{\circ}$ 200, 2009, p. 91-100.

23. Jean-Pierre Tuquoi, Paris-Alger, couple infernal, Grasset \& Fasquelle, 2007, 123 p.

24. William B. Cohen, "The Algerian War and the Revision of France's Overseas Mission”, French Colonial History, 4, 2003, pp. 227-239.

25. Benjamin Stora, Histoire de la guerre d'Algérie (1954-1962), quatrième édition, La Découverte, 2006, 130 p., p. 3.

26. Ibidem, p. 4.

27. Benjamin Stora, Histoire de l'Algérie coloniale (1830-1954), nouvelle édition, La Découverte, 2004, 124 p., p. 19.

28. Ibidem, p. 20.

29. Les développements sur la Corée du présent article sont inspirés des différents travaux sur le sujet que j'ai publiés par le passé. Voir Lionel Babicz, Le Japon face à la Corée à l'époque Meiji, Maisonneuve et Larose, 2002, 271 p.; «Japon, Chine, Corée: vers une conscience historique commune?», Ebisu, n 37, 2007, p. 19-46.

30. Benjamin Stora, Histoire de l'Algérie coloniale (1830-1954), op. cit., p. 18-19.

31. Ibidem, p. 20 et p. 30-34.

32. Edward I-te Chen, "The Attempt to Integrate the Empire: Legal Perspectives", in Ramon H. Myers and Mark R. Peattie (dir.), The Japanese Colonial Empire, 1895-1945, Princeton University Press, 1984, 560 p., pp. 240-274.

33. Chulwoo Lee, "Modernity, Legality, and Power", in Gi-Wook Shin and Michael Robinsons (dir.), Colonial Modernity in Korea, Harvard Univ. Asia Center, 1999, 496 p.

34. Edward I-te Chen, "The Attempt to Integrate the Empire: Legal Perspectives", op. cit., p. 273.

35. Jeffrey P. Bayliss, "Minority Success, Assimilation, and Identity in Prewar Japan: Pak Chungŭm and the Korean Middle Class", The Journal of Japanese Studies, 34.1, 2008, pp. 33-68.

36. Benjamin Stora, Histoire de l'Algérie coloniale (1830-1954), op. cit., p. 103-104.

37. Les origines de la colonisation japonaise en Corée sont plus anciennes, et remontent au moins au lendemain de la guerre sino-japonaise de 1894-95. La Corée était un protectorat japonais depuis 1905. Cependant, la colonisation change totalement de dimension avec l'annexion de 1910. 38. Benjamin Stora, Histoire de l'Algérie coloniale (1830-1954), op. cit., p. 85-86. Voir également plus haut les remarques sur le film Hors-la-loi.

39. Benjamin Stora, «La guerre des mémoires », Maghreb Machrek, nº 197, 2008, p. 13-18.

40. Ibidem, p. 14.

41. Ibid., p. 14-15.

42. Jean-François Daguzan, «Les relations franco-algériennes ou la perpétuelle leçon de tango », op. cit., p. 92.

43. Mis à part son emploi par le gouvernement français au début des hostilités, puis en 1960 par l'écrivain Jules Roy. Benjamin Stora, « La guerre des mémoires », op. cit., p. 13-14.

44. Journal officiel de la République française, $n^{\circ} 244$ du 20 octobre 1999, p. 15647.

45. Claude Liauzu (dir.), Dictionnaire de la colonisation française, Larousse, 2007, p. 322, introduction à l'article « Guerre d'Algérie : une guerre qui ne dit pas son nom ».

46. Benjamin Stora, La gangrène et l'oubli : la mémoire de la guerre d'Algérie, La Découverte, 1991, $376 \mathrm{p}$.

47. Ibidem, p. 9.

48. Mis à part quelques investissements économiques limités.

49. Jean-François Daguzan, "Les rapports franco-algériens, 1962-1992. Réconciliation ou conciliation permanente ? ", Politique étrangère (4), 1993, p. 885-896. Le développement suivant est largement inspiré de cet article. 
50. Ibidem, p. 891.

51. Kim Daejung (1925-2009) a été président de la Corée du Sud de 1998 à 2003.

52. La peine devait être commuée en vingt ans de prison, puis Kim Daejung fut finalement expulsé vers les États-Unis.

53. 日韓共同宣言 -21 世紀に向けた新たな日韓パートナーシップ-, 21世紀に向けた新たな日韓 パートナーシップのための行動計画

54. Voir les articles d'Arnaud Nanta et Samuel Guex, dans ce numéro.

55. Un échec similaire eut lieu en 2010 lors du centenaire de l'annexion de la Corée. Voir développement plus haut.

56. Hyeran Jo and Jongryn Mo, "Does the United States Need a New East Asian Anchor? The Case for U.S.-Japan-Korea Trilateralism", Asia Policy, no 9, 2010, pp.67-99; Victor Cha, Alignment Despite Antagonism: The United States-Korea-Japan Security Triangle, Stanford University Press, 2000, $373 \mathrm{p}$.

57. Jean-François Daguzan, « Les relations franco-algériennes ou la perpétuelle leçon de tango », op. cit., p. 94.

58. Ibidem, p. 96.

59. Ibid., p. 97.

60. « Sétif, 8 mai 1945 » : les prémices d'une repentance, Jeune Afrique, le 8 mai 2005.

61. Jean-François Daguzan, «Les relations franco-algériennes ou la perpétuelle leçon de tango », op. cit., p. 98.

62. Voir plus haut.

63. Journal officiel de la République française, 46, 24 février 2005.

64. Le Monde, 25 mai 2005.

65. « La loi qui fait débat », RFI.

66. Benjamin Stora, Thierry Leclère et Armelle Enders, La guerre des mémoires : la France face à son passé colonial - Entretiens avec Thierry Leclère, Éditions de l'Aube, 2008, 112 p., p. 19.

67. François Gèze et Gilles Manceron, "L'éloge de la colonisation est de retour ", Le Monde, 10 novembre 2010.

68. Benjamin Stora, La gangrène et l'oubli, op. cit.

69. Benjamin Stora, «La guerre des mémoires », op. cit., p. 13.

70. Christelle Taraud, La colonisation, Le Cavalier bleu, 2008, 126 p.

\section{RÉSUMÉS}

Cet article constitue une réflexion comparative sur les colonialismes japonais en Corée et français en Algérie, et sur les relations postcoloniales au sein de ces deux couples. La proximité de deux récents anniversaires - en 2010 le centenaire de l'annexion de la Corée par le Japon, et en 2012 le cinquantenaire de l'indépendance de l'Algérie - vient souligner la pertinence d'une analyse rapprochant les deux situations. Par bien des aspects, le colonialisme japonais en Corée était motivé par des idées similaires à la politique menée par les Français en Algérie. La Corée, tout comme l'Algérie, faisait partie intégrante du territoire national, et les deux colonies étaient destinées, à terme, à être totalement assimilées à la métropole. Aujourd'hui, les couples francoalgérien et nippo-coréen constituent deux variantes d'une même situation postcoloniale. Les relations en leur sein sont déterminées par des facteurs géostratégiques et des choix politiques. 
Cependant, la mémoire du passé colonial, même si elle est souvent instrumentalisée, ombrage fortement ces liens et témoigne de l'existence de plaies encore vives.

This article is a comparative reflection on Japanese colonialism in Korea and French colonialism in Algeria, and on the postcolonial relations within these two couples. The proximity of two recent anniversaries (in 2010 the centenary of the annexation of Korea by Japan, and in 2012 the fiftieth anniversary of the independence of Algeria) underlines the relevance of such a comparative approach. In many ways, Japanese colonialism in Korea was motivated by ideas similar to policies pursued by the French in Algeria. Both Korea and Algeria were defined as an integral part of the national territory, and both colonies were destined to be ultimately assimilated. Today, the French-Algerian and Japanese-Korean couples are two variants of a same postcolonial condition. The relations are determined by geopolitical factors and policy choices. However, the memory of the colonial past, although often exploited for political purposes, is still casting its shadow on these relations and reflects the existence of wounds still alive.

\section{INDEX}

Index géographique : Corée, Algérie, France, Japon

Mots-clés : colonialisme, post-colonialisme, relations nippo-coréennes, relations franco-

algériennes

Keywords : Korea, History, Colonialism, Post-colonialism, Japan-Korea Relations, France-Algeria Relations, Japon, France, Algeria

Thèmes : histoire 